\title{
ARTICLE OPEN Defining subpopulations of differential drug response to reveal novel target populations
}

\author{
Nirmal Keshava ${ }^{1,9}$, Tzen S. Toh ${ }^{2,3,9}$, Haobin Yuan ${ }^{4}$, Bingxun Yang ${ }^{4}$, Michael P. Menden ${ }^{5,6,7 *}$ and Dennis Wang $\mathbb{D}^{3,4,8 *}$
}

Personalised medicine has predominantly focused on genetically altered cancer genes that stratify drug responses, but there is a need to objectively evaluate differential pharmacology patterns at a subpopulation level. Here, we introduce an approach based on unsupervised machine learning to compare the pharmacological response relationships between 327 pairs of cancer therapies. This approach integrated multiple measures of response to identify subpopulations that react differently to inhibitors of the same or different targets to understand mechanisms of resistance and pathway cross-talk. MEK, BRAF, and PI3K inhibitors were shown to be effective as combination therapies for particular BRAF mutant subpopulations. A systematic analysis of preclinical data for a failed phase III trial of selumetinib combined with docetaxel in lung cancer suggests potential indications in pancreatic and colorectal cancers with KRAS mutation. This data-informed study exemplifies a method for stratified medicine to identify novel cancer subpopulations, their genetic biomarkers, and effective drug combinations.

npj Systems Biology and Applications (2019)5:36

; https://doi.org/10.1038/s41540-019-0113-4

\section{INTRODUCTION}

Drug developers face a conundrum in predicting the efficacy of their investigational compound compared to existing drugs used as the standard of care treatment. Systematic screening of drug compounds across a variety of genomic backgrounds in cancer cell lines has improved clinical trial design and personalised treatments. ${ }^{1}$ Following the pioneering $\mathrm{NCl}-60$ screen comprising 59 unique cell lines, ${ }^{2}$ modern high-throughput screens such as the Genomics of Drug Sensitivity in Cancer (GDSC), ${ }^{3,4}$ the Cancer Cell Line Encyclopaedia (CCLE), ${ }^{5}$ and the Cancer Therapeutics Response Portal (CTRP) ${ }^{6-8}$ have characterised $>1000$ cancer cell lines with the goal of establishing the genetic landscape of cancer. The deep molecular characterisation of these large cell line panels is complemented with high-throughput drug screens, which enables the discovery of drug response biomarkers. For example, analysis of the generic BRAF inhibitors PLX4720 (progenitor of vemurafenib), SB590885, and $\mathrm{Cl}-1040$ reproduced drug sensitivity association with BRAF mutation in melanoma, or afatinib sensitivity with ERBB2 amplifications in breast cancer. ${ }^{3,4,9}$ These associations between genetic variants and treatment response have helped identify specific patient subpopulations who are most likely to benefit from treatment. In Phase III clinical trials, however, for new drugs to be successful, they must demonstrate a significant improvement over the existing standard of care. Accurately defining in which subpopulations a new drug demonstrates improved differential efficacy over other drugs targeting the same disease could lead to both better clinical outcomes as well as new targeted therapies.

While several methods have been proposed to identify drug response biomarkers in cell lines for precision medicine and drug repositioning, $4,5,10,11$ there is a need for more objective and unsupervised approaches for identifying subpopulations with differences in drug response (differential drug response), and consequently, systematically gain mechanistic insights from biomarkers. Most approaches capable of comparing multiple drugs measure the overall similarity (or correlation) based on a single response summary metric, ${ }^{7,12}$ which permits drug repositioning based on subpopulations with similar behaviour, but neglects ones that behave differently (Supplementary Fig. S1a). Here, we used an unsupervised technique to identify the perimeters of differentially sensitive or resistant subpopulations and which may be generalised to stratify the pharmacology response for any pair (or n-tuple) of targets using any number of drug response summary metrics (e.g. IC 50 or AUC). Segmentation of the overall population occurs top-down and along globally optimal contours that are derived explicitly and maximise the differences between the two resulting subpopulations. The segmentation continues recursively and is modulated by multiple user-defined criteria such as the size or separability of the resulting subpopulations. Higher threshold values for both result in less granular subpopulations but increase certainty that the subpopulations and the quantities estimated from them are both distinct and accurate.

We present results from our platform, SEABED (SEgmentation And Biomarker Enrichment of Differential treatment response), to demonstrate how unsupervised machine learning can discover intrinsic partitions in the drug response measurements of two or more drugs that directly correspond to distinct pharmacological patterns of response with therapeutic biomarkers. Addressing the challenges in comparing the response of two drugs, SEABED initially assesses two gold standards with established clinical biomarkers, namely the differential response of a BRAF inhibitor and MEK inhibitor with anticipated BRAF and KRAS mutations, ${ }^{13-16}$ and an EGFR inhibitor and MEK inhibitor with expected biomarkers of EGFR, ERBB2, and KRAS mutations. ${ }^{17-20}$ Next, we systematically compare how different drugs targeting the MAPK and PI3K-AKT pathway yield different patterns of response within

\footnotetext{
${ }^{1}$ Constellation Analytics, LLC., Needham, MA, USA. ${ }^{2}$ The Medical School, University of Sheffield, Sheffield S10 2 RX, UK. ${ }^{3}$ Sheffield Institute for Translational Neuroscience, University of Sheffield, Sheffield S10 2HQ, UK. ${ }^{4}$ Department of Computer Science, University of Sheffield, Sheffield S1 4DP, UK. ${ }^{5}$ Institute of Computational Biology, Helmholtz Zentrum München—German Research Center for Environmental Health, 85764 Neuherberg, Germany. ${ }^{6}$ Department of Biology, Ludwig-Maximilians University Munich, 82152 Martinsried, Germany. ${ }^{7}$ German Centre for Diabetes Research (DZD e.V.), 85764 Neuherberg, Germany. ${ }^{8}$ NIHR Sheffield Biomedical Research Centre, Sheffield S10 2HQ, UK. ${ }^{9}$ These authors contributed equally: Nirmal Keshava, Tzen S. Toh. *email: michael.menden@helmholtz-muenchen.de; dennis.wang@sheffield.ac.uk
} 
subpopulations. We show how differential drug response may indicate benefit for drug combinations explained through independent action rather than probable synergy by examining subpopulations uniquely sensitive to a single drug, ${ }^{21}$ which may be precisely targeted by identified biomarkers. This is particularly evident in subpopulations of cell lines with divergent response, where there is sensitivity to either drug but not both. Finally, we demonstrate how the analysis of differential response can guide the design of clinical trials by revealing specific indications where an investigational therapy may be more effective than the standard treatment.

\section{RESULTS}

We applied our technique to discover subpopulations of cell lines in which two or more compounds, possibly addressing the same disease state or even targeting the same genetic alteration, have a common pharmacological pattern of response. By further associating enriched genetic alterations in subpopulations with specific patterns of response, we shed light into molecular mechanisms responsible for patient subpopulations that respond differently to two drugs.

\section{Identifying subpopulations of differential drug response}

We first considered the specific circumstance in which two drugs engage different targets within the same signalling pathway, namely agents targeting MAPK signalling. SEABED analysed $>800$ cancer cells for each pair of drugs, and we evaluated two established drug response measures: the drug concentration required to reduce cell viability by half $\left(\mathrm{IC}_{50}\right)$ and the area under the dose-response curve (AUC; Fig. 1a). SEABED employed a multivariate similarity measure to compare the vector patterns of response for each distinct pair of cell lines without requiring a priori assumptions on the number or distribution of the subpopulations. The result is a diverse cell line population segmented into distinct subpopulations having homogeneous patterns of drug response (Fig. 1b). Here exemplified, we show that the drug response of 802 cell lines treated with either SB590885 (BRAF inhibitor) or Cl-1040 (MEK inhibitor) could be segmented into seven distinct subpopulations with a median size of 40 cell lines by integrating the two metrics of drug response, AUC and $\mathrm{IC}_{50}$ (Fig. 1C; see Supplementary Figs. S1b and S1C for individual cell lines segmented by $\mathrm{IC}_{50}$ and $A U C$, respectively). We comprehensively evaluated pan-cancer somatic events to nominate biomarkers, and found that the subpopulation sensitive to both inhibitors was significantly enriched for BRAF mutants $(P=$ 3.87e-14, hypergeometric test), while another subpopulation was exclusively sensitive to the MEK inhibitor and significantly enriched for KRAS mutations ( $P=0.00589$, hypergeometric test).

In another example we examined a case where one inhibitor might overcome resistance to another inhibitor targeting the same pathway: AZD6244/ARRY-142886 selumetinib (MEK inhibitor) with afatinib (EGFR and ERBB2 dual inhibitor) across 839 cell lines (Fig. 1d). Strong markers of sensitivity for selumetinib are subpopulations carrying known associated KRAS, NRAS, and BRAF mutations (Fig. 1d, e). A less anticipated association is APC loss-offunction sensitivity to selumetinib, albeit this was also found with trametinib (another MEK inhibitor) in APC-deficient mice. ${ }^{22}$ We reproduced the well-established associations of afatinib with either EGFR and ERBB2 amplifications, ${ }^{4,23}$ and surprisingly our unsupervised segmentation returned two subpopulations enriched for EGFR amplifications. The more sensitive subpopulation is solely enriched for EGFR amplifications, while the less sensitive subpopulation additionally includes activating PIK3CA mutations. In concordance with recent literature, PI3K-AKT signalling drives acquired drug resistance to EGFR inhibitors in lung cancer. $^{24}$
Drug response segmentation resulted in 14 subpopulations with a median size of 37 (Fig. 1d). The subpopulation enriched for EGFR, ERBB2, and PI3KCA mutations has an average $\log \left(\mathrm{IC}_{50}\right)$ of $1.01 \mu \mathrm{M}$ for selumetinib and $-0.672 \mu \mathrm{M}$ for afatinib. In contrast, the $B R A F$ mutation was enriched in a subpopulation where the average $\log \left(\mathrm{IC}_{50}\right)$ for selumetinib was $-1.097 \mu \mathrm{M}$ and $0.625 \mu \mathrm{M}$ for afatinib. The difference in response between afatinib and selumetinib was significantly greater ( $t$-test $P<0.01)$ between the subpopulations identified and the total population of PIK3CA or BRAF mutant cell lines (Fig. 1f, g).

Cross-comparison of multiple drugs redefines best-in-class drugs for specific subpopulations

Although there is a larger portfolio of clinical drugs with identical putative targets, their responses may differ substantially in subpopulations as a consequence of multiple factors, for example mode-of-action, different off-target effects, and binding properties. The ability to discover cell line subpopulations with distinct pharmacological patterns of response characterised by genetic mutations re-defines best-in-class drugs by their differential response to other drugs in a specific subpopulation, rather than their absolute response across an entire population.

In order to demonstrate this approach for drug discovery, we applied SEABED to 745 cell lines across cancer types to evaluate the differential response in those cell lines to five inhibitors $(\mathrm{Cl}-$ 1040, PD0325901, RDEA119, selumetinib, and trametinib) which all target the MEK protein (Fig. 2a). The segmentation of cell lines revealed 13 subpopulations with different patterns of response and 3 having enriched biomarkers (Supplementary Fig. S2a). Two subpopulations were sensitive to all MEK inhibitors, with trametinib achieving the greatest sensitivity. In one subpopulation, the KRAS mutation was enriched (Fisher exact $p$ value $=$ $1.12 \mathrm{e}-4$ and $40.8 \%$ of the cell lines) while another had the BRAF mutation enriched (Fisher exact $p$ value $=1.39 \mathrm{e}-7$ and $50 \%$ of the cell lines). In contrast, another subpopulation was enriched with the $R B 1$ mutation (Fisher exact $p$ value $=3.84 \mathrm{e}-2$ and $21.6 \%$ of cell lines), within which the cell lines were almost uniformly resistant to all MEK inhibitors.

Distribution of subpopulations highlight distinct pharmacological relationships between PI3K-AKT and MAPK signalling

Next, we used SEABED to investigate the cross-talk between two frequently active cancer pathways, MAPK and PI3K-AKT signalling, by systematically comparing pairs of drugs targeting different genes of each pathway (Fig. 2). In total, SEABED performed 324 pairwise comparisons of 18 PI3K-AKT and 18 MAPK pathway inhibitors. Each drug pair was classified into five categories based on the distribution of subpopulation responses: (i) no preferential response, (ii) sensitive to both MAPK and PI3K-AKT pathway inhibitors (Supplementary Fig. S2b), (iii) greater sensitivity to MAPK pathway inhibitors (Supplementary Fig. S2c), (iv) greater sensitivity to PI3K-AKT pathway inhibitors (Supplementary Fig. S2d), (v) each subpopulation was sensitive to either a MAPK pathway inhibitor or a PI3K-AKT pathway inhibitor but not both, i.e. divergent response (Supplementary Fig. S2e). Common enriched mutations and cancer tissue types found across these examples include $B R A F$ and $R B 1$ mutations as well as skin, small-cell lung cancer, lymphoma, and leukaemia tissue types, respectively (Supplementary Figs. S2b-e). An inactive MAPK pathway based on gene expression profiles was found in a subpopulation sensitive to KIN001-102 (AKT inhibitor) and resistant to trametinib (MEK inhibitor) (Supplementary Fig. S2d). MAPK pathway inactivity was also found within a subpopulation sensitive to ZSTK474 (PI3K inhibitor) but resistant to RDEA119-2 (MEK inhibitor). The enriched MAPK-PI3K and TNFa pathway expression markers showed inactivity in a subpopulation resistant 
a
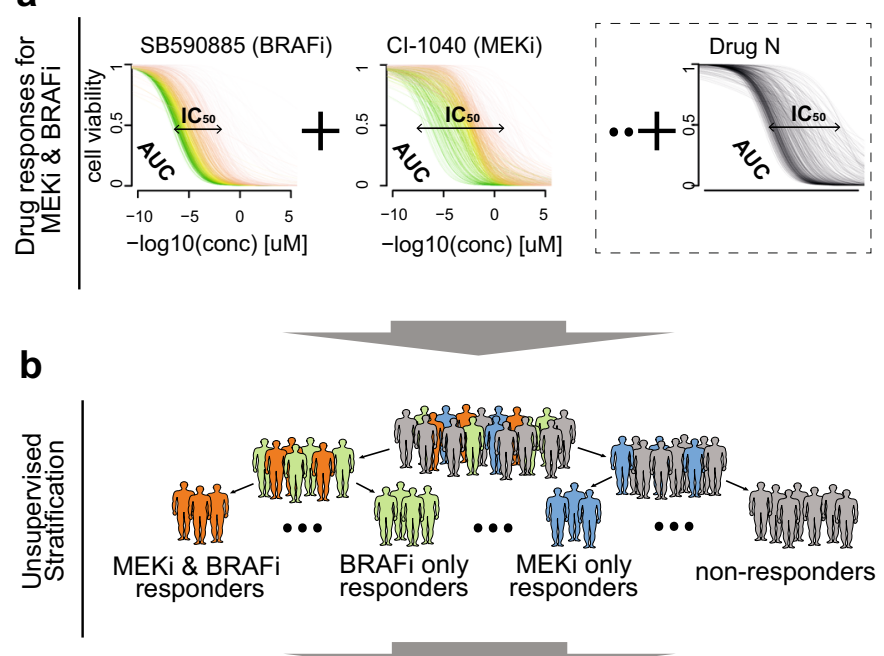

C

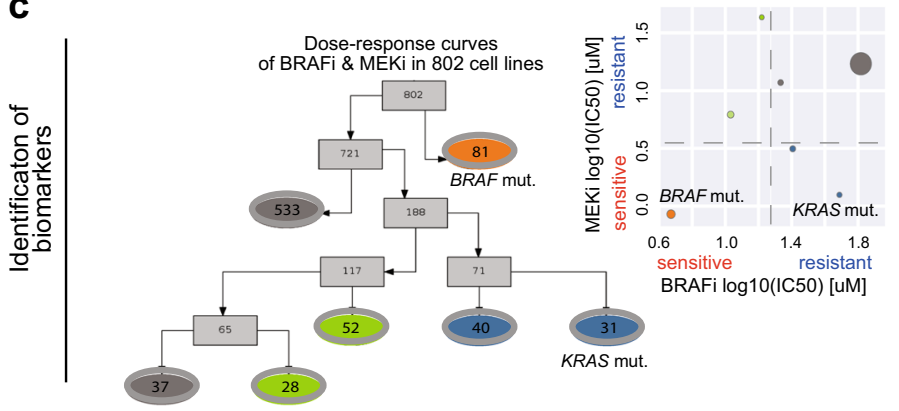

e
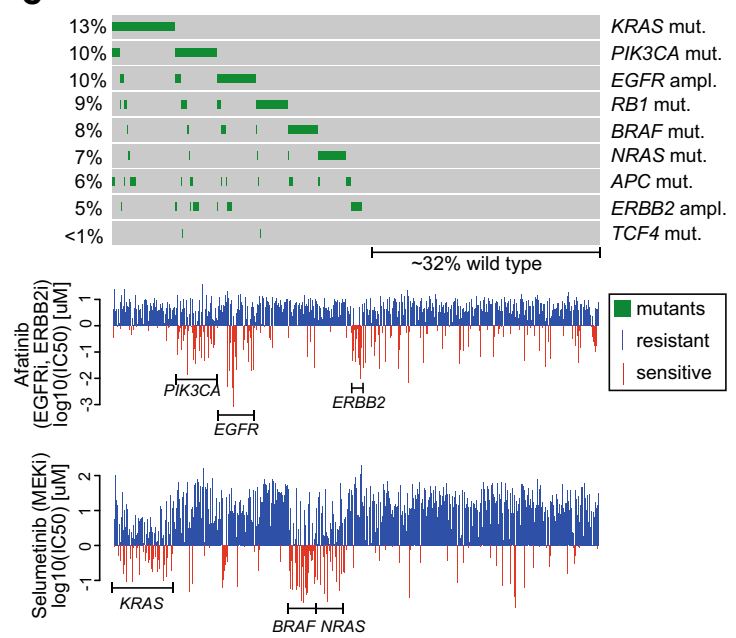

d

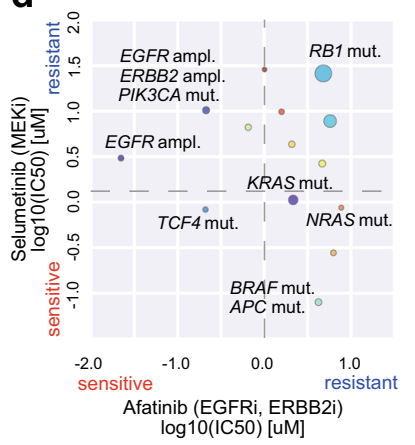

Fig. 1 Segmentation of a population based on pharmacological patterns of response discovers subpopulations with differential response. a Dose response curves of two or more drugs are measured across a population of just over 1000 cancer cell lines. $\mathbf{b}$ The population is segmented into distinct and homogeneous subpopulations based on their response to multiple drugs. When comparing two drugs, subpopulations can be categorised based on their mean $\log \left(\mathrm{IC}_{50} \mathrm{~s}\right)$ : sensitive to both drugs (orange), sensitive to drug $A$ but not drug $B$ (green), sensitive to drug B but not drug A (blue), and resistant to both drugs (grey). c Segmentation results for a BRAF inhibitor (SB590885) and a MEK inhibitor (Cl-1040). Tree nodes contain the number of cell lines and are coloured based upon their category of response. Significance testing of 735 cancer functional events reveals subpopulations enriched for BRAF and KRAS mutations. d Scatter plot showing derived subpopulations based on their pharmacological responses for afatinib and selumetinib. Dashed lines indicate 20th percentile of log $\left(I_{50}\right)$ values for each drug. PIK3CA, EGFR, ERBB2, KRAS, NRAS, BRAF, APC, TCF4, and RB1 mutations were found enriched in the associated subpopulations. e OncoPrint visualising the percentage of mutations of selected genes in cell line panel treated with either afatinib (EGFR inhibitor) or selumetinib (MEK inhibitor). The waterfall plots comparing the response of the cell lines to afatinib and to selumetinib. $f$ Boxplot of difference in $\log \left(\mathrm{IC}_{50}\right)$ values between afatinib and selumetinib response for wild-type cell lines, all cell lines with $P I 3 K C A$ mutation, and cell lines in derived subpopulations with enriched PI3KCA mutation. $\mathbf{g}$ Same as panel $\mathbf{f}$, but for BRAF mutations

to both RDEA119-2 and ZSTK474 (Supplementary Fig. S2e). While this approach classified the distribution of subpopulations based on somewhat arbitrary thresholds for sensitivity, we also measured the weighted average Pearson correlation across subpopulations to identify interesting drug pairs (Supplementary Fig. S3).

We found 20 drug pairs subpopulations with sensitivity to both PI3K-AKT and MAPK pathway inhibition. This association between subpopulation size and sensitive response was significant when comparing a CRAF inhibitor (TL-2-105) to PI3K-AKT signalling inhibitors (hypergeometric test $P=2.358 \mathrm{e}-5$ ). The same trend was observed for inhibiting ERK (FR-180204) or RSK (FMK) compared to inhibiting any PI3K-AKT signalling gene $(P=0.0185$ and $P=$ $2.358 \mathrm{e}-5$, respectively; hypergeometric test), but interestingly there was no mutual sensitivity when comparing to BRAF inhibitors and only two cases for MEK inhibitors.

There were 20 drug pairs with a significantly high proportion of subpopulations $(P<0.05)$ exhibiting greater sensitivity to MAPK pathway inhibition. This phenotype is strongly pronounced in pairs with BRAF inhibitors (hypergeometric test $P=0.0166$ ). Twenty drug pairs were found with significantly high proportions of preferential PI3K-AKT pathway inhibition. This is observed when comparing MEK inhibitors ( $\mathrm{Cl}-1040$ and selumetinib- 1$)$ to PI3K-AKT signalling inhibitors $(P=0.0185$ and $P=0.00273$, respectively; hypergeometric test).

In 22 cases, we observed drug pairs with each subpopulation having sensitivity to either an MAPK pathway or an PI3K-AKT pathway inhibitor but not both, i.e. divergent response. This response type was enriched for pairs of any PI3K-AKT pathway inhibitors and BRAF (PLX4720-2) or MEK inhibitors $(P=0.026$ and $P=0.0135$, respectively; hypergeometric test), and even significant for PI3K inhibitors in comparison with either the EGFR (erlotinib) or MEK inhibitors ( $P=0.0315$ and $P=0.0173$; hypergeometric test). Response patterns for all drug pairs can be explored in our portal (Supplementary Website S1; https://szen95.github.io/ SEABED). 
a

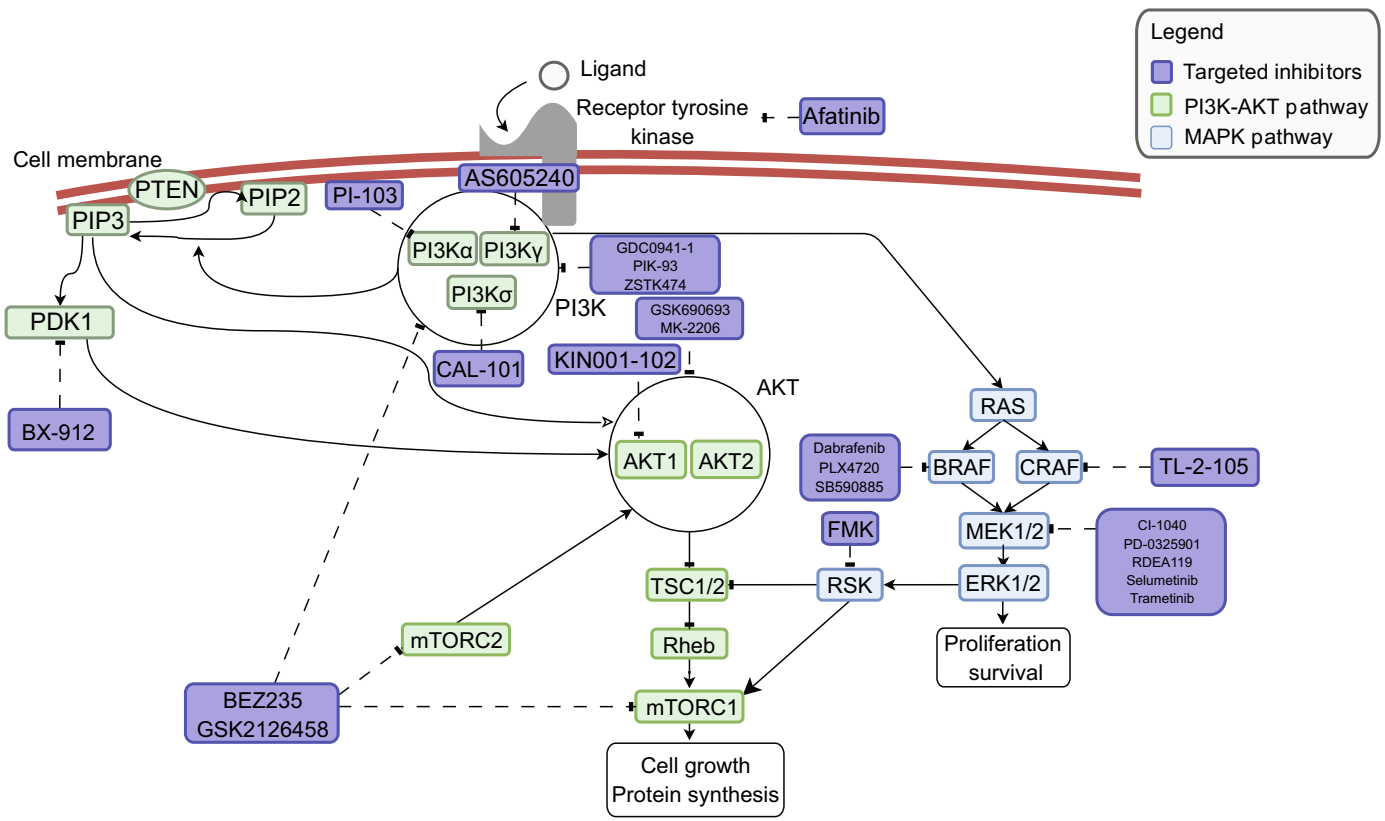

b

Comparing drugs based on distribution of subpopulations
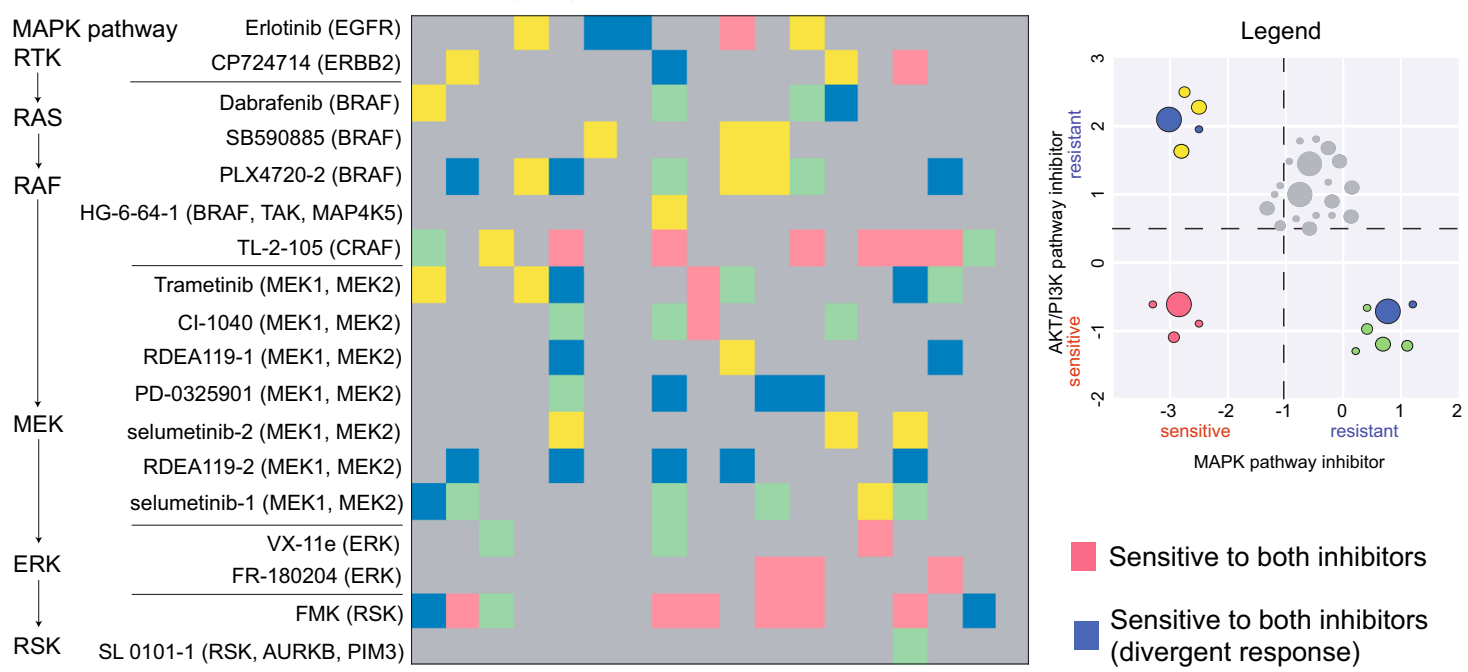

Sensitive to both inhibitors

Sensitive to both inhibitors (divergent response)

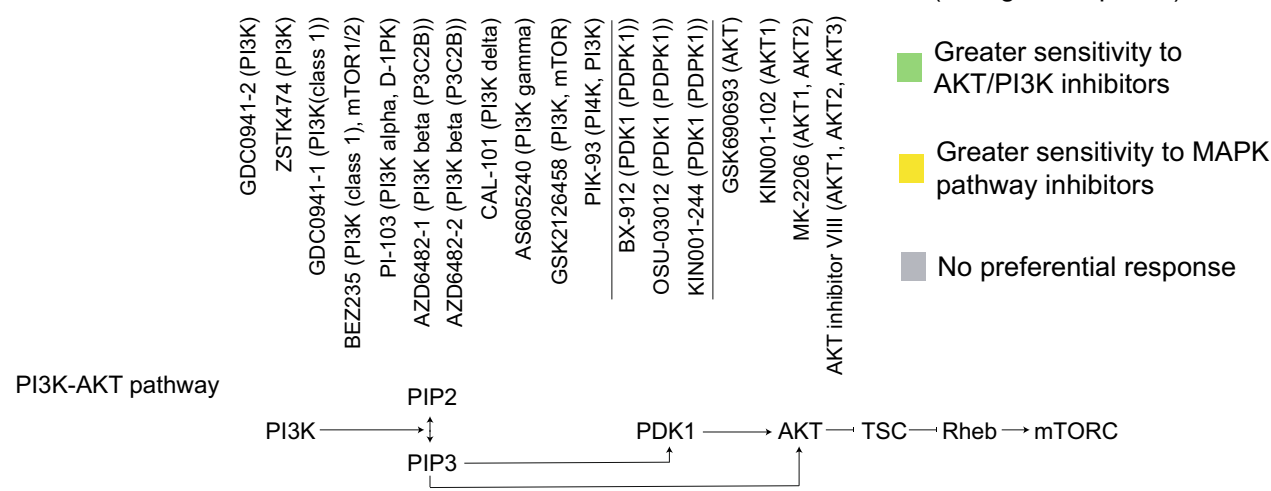

Subpopulations of differential response identifies drug combination efficacy

Previous studies have hypothesised that the efficacy of many approved drug combinations can be explained by the independent action of single agents on different patient subpopulations with cancers driven by multiple pathways. ${ }^{21}$ We hypothesised that
SEABED comparisons of drug pairs would highlight subpopulations of differential response that would exhibit synergistic or independent action effects when the drugs are tested in combination. Additionally, our method enables us to explore putative biomarkers of such populations. Systematic comparison of responses between two drugs highlighted subpopulations of 
Fig. 2 Distinct drug response types after unsupervised segmentation of pharmacological response pattern for targeting MAPK or PI3K-AKT signalling. a MAPK and PI3K-AKT pathways illustrating drugs in purple boxes which were assessed, and their different gene targets in the pathway. Genes in the green boxes are involved in the PI3K-AKT pathway while genes in the blue boxes are involved in the MAPK pathway. b Heatmap illustrating 324 pairwise comparison of responses for 18 different inhibitors targeting the MAPK pathway and 18 different inhibitors targeting the PI3K-AKT pathway. The 20th-percentile of $\log \left(\mathrm{IC}_{50}\right)$ values for each drug was determined based on the distribution of log $\left(\mathrm{IC}_{50}\right)$ values across all cell lines after SEABED segmentation. Based on these 20th percentile cutoffs, we assessed whether there was overrepresentation of subpopulations in each quadrant and categorised each drug pair into five categories: (i) no preferential response (grey), (ii) subpopulations sensitive to both MAPK and PI3K-AKT pathway inhibitors (pink), (iii) greater MAPK pathway sensitivity (yellow), (iv) greater PI3K-AKT pathway sensitivity (green), or (v) sensitive to either inhibitor but not both (divergent response, blue)

cell lines in which there was sensitivity to either drug but not both (divergent response). We observed this phenomenon in 22 drug pair comparisons, including an MEK inhibitor (RDEA119-2) which showed divergent responses to four PI3K inhibitors (PI-103, GSK2126458, ZSTK474, and CAL-101; Fig. 3a; Supplementary Fig. S4a-d, respectively). TNFa pathway inactivity was found in a subpopulation resistant to both drugs in three out of these four drug pairs. MAPK pathway inactivity was instead found in subpopulations resistant to the MEK inhibitor but sensitive to the PI3K inhibitor (Fig. 3a, Supplementary Figs. S4a-d). Drug pairs with divergent response were also observed in cell lines treated with PLX4720-2 (BRAF inhibitor) and two PI3K inhibitors (PI-103 and ZSTK474; Fig. 3b, Supplementary Figs. S4e and S4f). Cancer tissue types found enriched in these two drug pairs included skin, leukaemia, and lymphoma tissue types. There was only one expressed pathway found in a subpopulation resistant to PLX4720-2 but sensitive to ZSTK474, which was the MAPK pathway expression marker indicating inactivity (Supplementary Fig. S4f). Two subpopulations with a high proportion of BRAF mutations were identified with greater sensitivity to the BRAF inhibitor (Fig. 3b). These two subpopulations had a combined total of 79 cell lines (Fig. 3c). The subpopulation with an average log $\left(\mathrm{IC}_{50}\right)$ of $-0.0232 \mu \mathrm{M}$ for PLX4720-2 and $0.771 \mu \mathrm{M}$ for PI-103 had 21 cell lines with $B R A F$ mutation $(87.5 \% ; P=9.75 \mathrm{e}-17)$. In contrast, the subpopulation with an average $\log \left({ } C_{50}\right)$ of $0.808 \mu \mathrm{M}$ for PLX4720-2 and $0.562 \mu \mathrm{M}$ for PI-103 had 22 cell lines with BRAF mutation (40.4\%; $P=5.12 \mathrm{e}-7$ ) (Fig. 3b). We observed 17 individual cell lines with BRAF mutation that are resistant to both drugs (Supplementary Fig. S4g).

We next examined the drug pairs as combination therapies in cell lines ${ }^{25}$ and patient-derived tumour xenograft models (PDXs) ${ }^{26}$ to investigate whether the drug pairs with divergent response and subpopulations with preferential sensitivity to one drug would be associated with efficacy of their combination treatment (Fig. 3d). SEABED first compared the single drug responses of BRAF, MEK, and $\mathrm{PI} 3 \mathrm{~K}$ inhibitors as before to identify BRAF mutant subpopulations with differential response. When the drugs were tested as combinations in BRAF mutant cell lines, the MEK/PI3K inhibitor combination had a surprising similar level of synergy as BRAF/MEK combinations, which was recently a clinically approved combination. ${ }^{27,28}$ Also surprising, these two combinations had significantly higher synergistic effect when used on BRAF mutant cell lines compared to all cell lines ( $t$-test $P=0.0204$ ), and compared to all drug combinations tested ( $t$-test $P=1.46 \mathrm{e}-5$; Fig. 3e; Supplementary Fig. S4h). In terms of overall efficacy in PDXs, we observed a similar level of inhibition to tumour volume for the BRAF/PI3K inhibitor combination on BRAF mutant cells when compared to the clinically approved BRAF/MEK combination and a significantly greater ( $t$-test $P=0.0418$ ) inhibition of tumour growth compared to all combinations (Fig. 3f; Supplementary Fig. S4i). Notably, previous work suggested in vivo efficacy of drug combinations is mostly driven by the monotherapy agents targeting independent mechanisms ${ }^{26}$; however, they did not exclude the possibility that such drug combinations may also be synergistic. Here, we highlight a drug combination example, where in vivo efficacy is driven by targeting independent mechanisms, and complementary being synergistic. This example highlights that both concepts, synergy and targeting independent mechanism may contribute to combination efficacy in patients.

\section{Lack of subpopulations of differential response may explain} clinical failure

Sometimes, despite strong preclinical evidence, some drugs do not succeed in clinical trials. ${ }^{29}$ One such trial was SELECT-1 (Supplementary Table S1), which compared the efficacy of combining selumetinib and docetaxel to docetaxel alone in patients with advanced KRAS-mutant non-small cell lung cancer (NSCLC). ${ }^{30}$ Although there were KRAS mutant cell lines sensitive to selumetinib in preclinical testing, ${ }^{31}$ we re-examined the pharmacological data with SEABED to assess whether there were distinct subpopulations that justified the patient selection criteria for KRAS mutation.

In this analysis, instead of only inspecting the subpopulation identified by SEABED when the segmentation algorithm terminated, we thoroughly examined all possible subpopulations. SEABED identified a total of 61 possible subpopulations from 840 cell lines across tissue types tested with selumetinib and docetaxel (Fig. 4a). Ten subpopulations were more sensitive to selumetinib than docetaxel (Fig. 4b), and five of those subpopulations were enriched for KRAS mutation. However, those subpopulations enriched for NSCLC KRAS mutants were small in size and mostly exhibited less sensitivity to selumetinib compared to docetaxel (Supplementary Figs. S5a, b). The distribution of different KRAS mutations (p.G12C vs p.G12V) was also no different in selumetinib sensitive subpopulations compared to resistant subpopulations (Supplementary Figs. S5c, d). Independent of mutation status, only $8.9 \%$ of NSCLC cell lines were found in subpopulations more sensitive to selumetinib, whereas $14.8 \%$ cell lines originating from aerodigestive cancer types (e.g. oesophageal) were found in these subpopulations (Supplementary Fig. S5e, f).

Next, we focused on subpopulation_60, which had the greatest difference in sensitivity ( $\mathrm{I}_{50}$ and $\mathrm{AUC}$ ) to selumetinib compared to docetaxel (Fig. 4c). This subpopulation of 122 cell lines was enriched in KRAS mutations $(28.8 \%, P=3.061 \mathrm{e}-4)$ found across multiple tissue types. NSCLC cell lines accounted for only $8 \%$ of this subpopulation, with $50 \%$ of those cell lines being KRAS mutants. Colorectal and pancreatic cell lines accounted for $15 \%$ and $8 \%$ respectively of the subpopulation, and they both had a higher proportion of KRAS mutations (56\% and $100 \%$, respectively; Fig. 4d).

\section{DISCUSSION}

The ability to identify distinct subpopulations based on multiple measures of drug response (e.g. $I_{50}$ and $A U C$ ) and extract their biomarkers is the basis for personalised therapeutics, which may ultimately increase the likelihood of successful clinical trials. ${ }^{32,33}$ Using a network-based segmentation algorithm coupled with biomarker detection (SEABED), we investigated well-established pharmacological targets and clinical biomarkers by comparing the response patterns for BRAF (SB590885) and MEK (Cl-1040) 
a

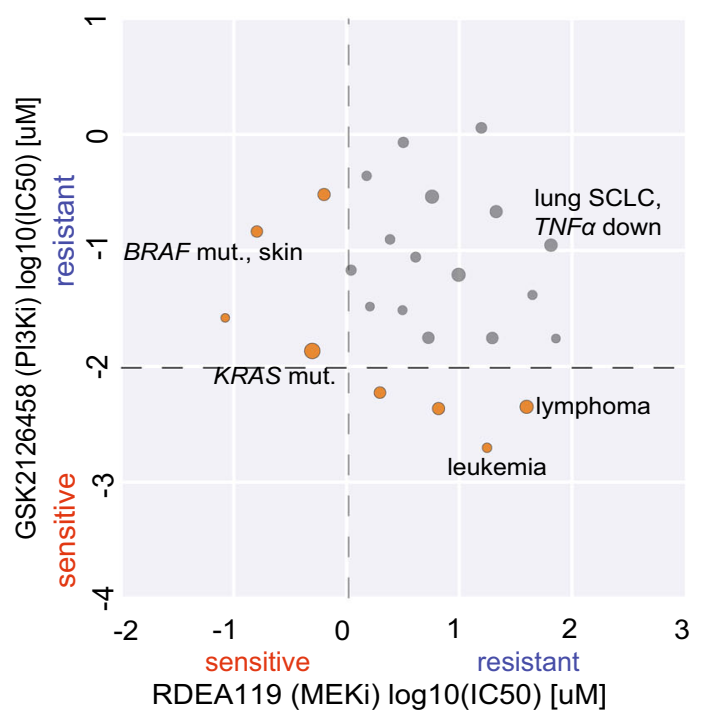

C

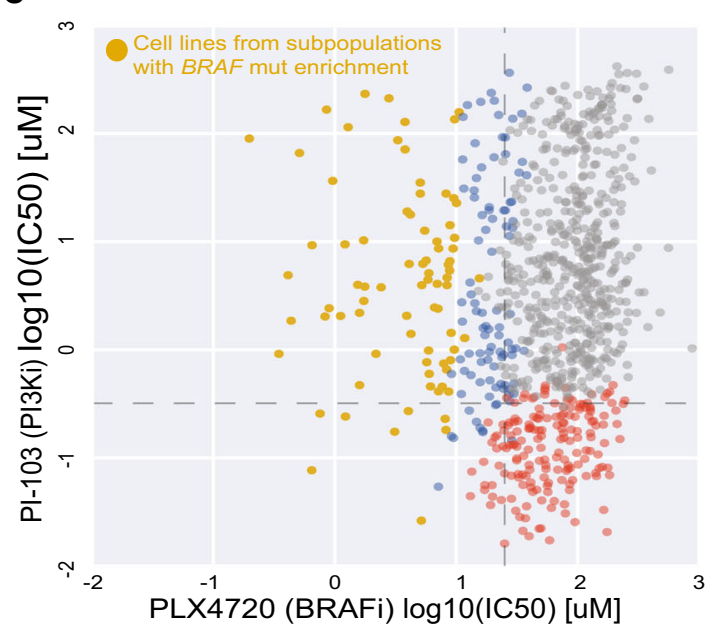

b

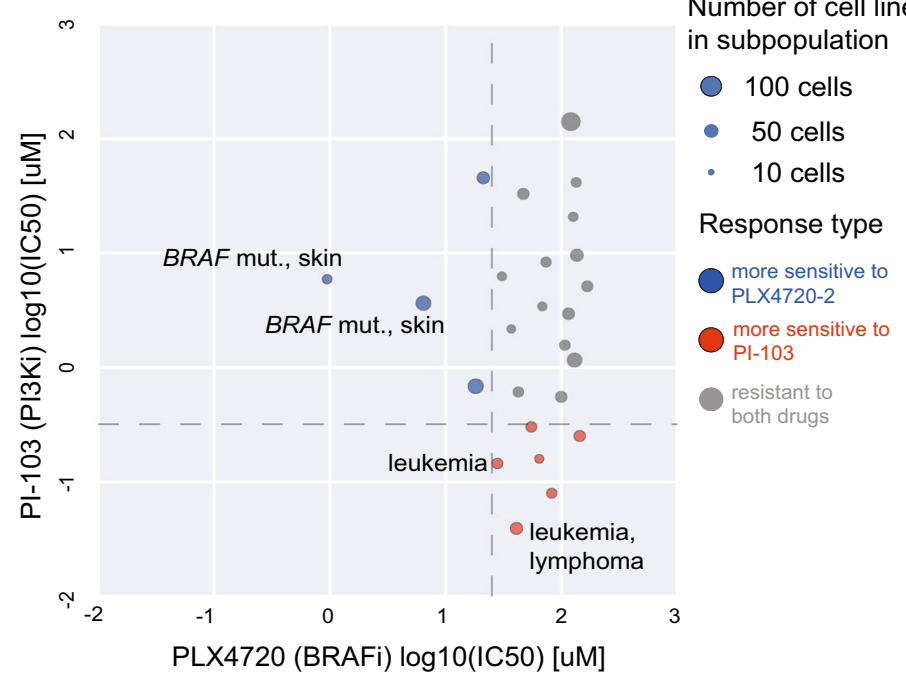

d

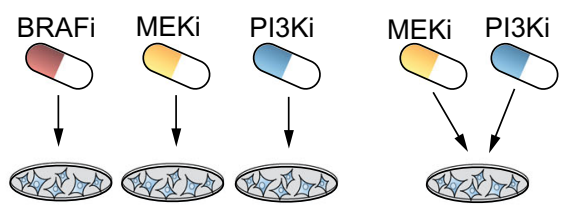

Cancer cell line

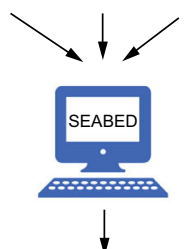

Divergent response
Number of cell lines in subpopulation

100 cells

50 cells

10 cells

Response type

e sensitive

$\mathrm{PI}-103$

resistant to both drugs
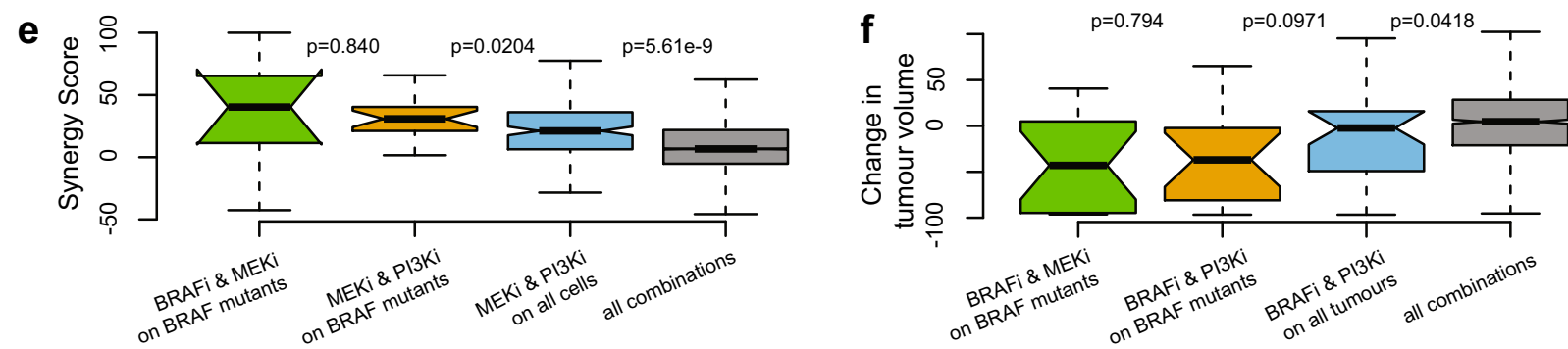

Fig. 3 Divergent response exemplified with PI3K inhibition in comparison to MEK and BRAF inhibitors. a Scatter plot showing subpopulations that exhibit divergent pharmacological response to RDEA119-2 (MEK inhibitor) and GSK2126458 (PI3K inhibitor). Dashed lines indicate 20th percentile of $\log \left(\mathrm{IC}_{50}\right)$ values for each drug. BRAF and KRAS mutations were found enriched in the associated subpopulations. Enriched mutations, cancer tissue types, and/or expression pathway markers are labelled beside each subpopulation. Enriched expression pathway markers represent either the activated or inactive pathways in the subpopulation. Each pathway was named "-up" for activated pathways or "-down" for inactive pathways. b Subpopulations from comparison of PLX4720-2 (BRAF inhibitor) and PI-103 (PI3K inhibitor) responses. c Individual cell line responses from PLX4720-2 and PI-103 coloured by the subpopulations they were grouped in. d Workflow illustrating cell lines being tested with individual inhibitors and their joint pharmacological patterns of response. Drug pairs with divergent response and $B R A F$ mutant subpopulations suggest a target for drug combination therapies, which are validated in cell lines ${ }^{25}$ and patient-derived tumour xenograft (PDX) models. ${ }^{26}$ e In vitro synergistic effect of combining MEK inhibitors with PI3K or BRAF inhibitors in all cell lines and just those with BRAF mutations. This was also compared to measured synergy for all combinations tested in cell lines. $\mathrm{f}$ In vivo effect of combining BRAF inhibitors with PI3K or MEK inhibitors. Measured response is the change in tumour volume following treatment. BRAF and MEK inhibitor combinations have also been shown to be effective in the patients ${ }^{28}$ 


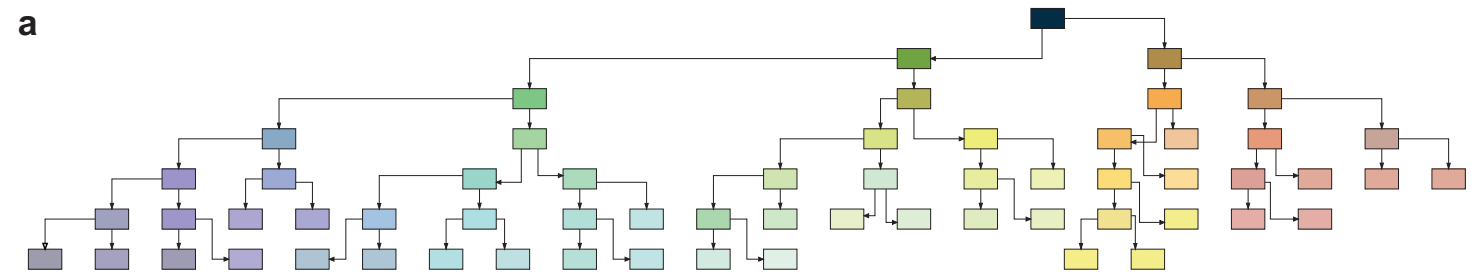

b
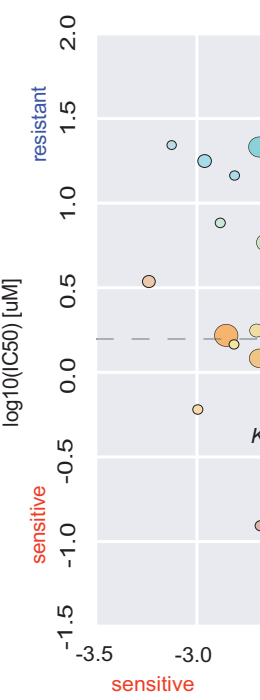

Number of cell lines

in subpopulation
Pan-cancer cell lines

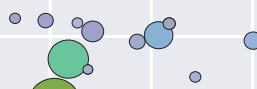

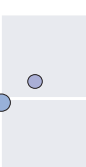

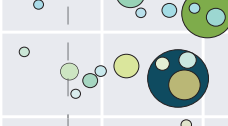

○

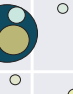

0
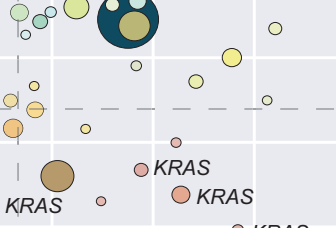

KRAS OKRAS

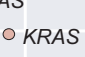

subpop. 60

○
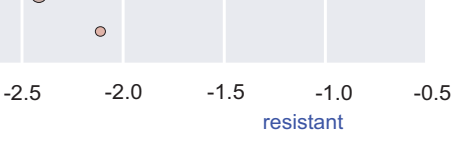

Docetaxel (Microtubules) $\log 10($ IC50) [uM]
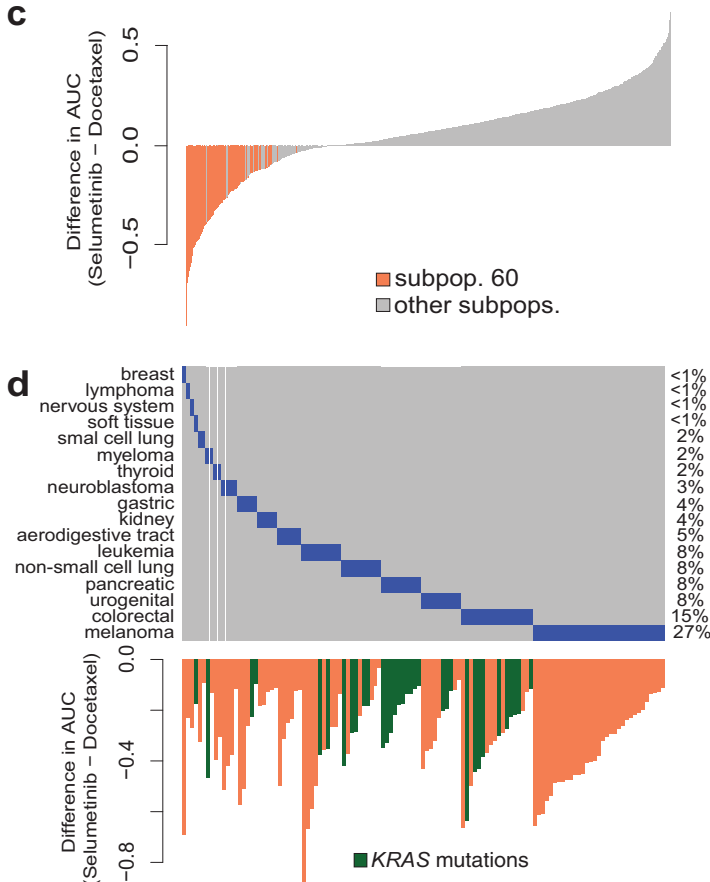

Fig. 4 Subpopulations of cell lines indicating differential response between docetaxel and selumetinib. a Tree diagram illustrating the segmentation process of 840 cell lines across cancer types into subpopulations based on their response to docetaxel and selumetinib. Branch colours distinguish subsets of subpopulations with darker colours indicating increasing number of cell lines in the subpopulation. b Scatter plot of subpopulations discovered through progressive segmentation based on average log $\left(\mathrm{IC}_{50}\right)$ values. Dashed lines indicate 20 th percentile of $\log \left(I C_{50}\right)$ values for each drug. The KRAS mutation is enriched in five subpopulations exhibiting sensitivity to selumetinib and resistance to docetaxel, including subpopulation 60. The colours of the subpopulations correspond to the location of the subpopulation in the tree diagram. c Bar plot illustrating the difference in AUC values for each cell line. The orange bars highlight the cell lines within subpopulation 60. d Heatmap showing the percentage of each cancer type enriched within the cell lines in subpopulation 60 . The bar plot illustrates the KRAS mutations within the cell lines that are highlighted in green

inhibition, which expectedly reproduced subpopulations sensitive to both drugs and enriched for BRAF mutants. ${ }^{34-36}$ In another example, SEABED compared EGFR/ERBB2 (afatinib) and MEK (selumetinib) inhibition to reveal expected biomarkers such as $B R A F, K R A S$, and NRAS mutations for selumetinib, ${ }^{13-16}$ and afatinib associated with EGFR and ERBB2 amplifications. ${ }^{37,38}$ Interestingly, the more afatinib-resistant subpopulation was enriched for PI3KCA-activating mutation, which may cause acquired resistance. ${ }^{24}$ When we systematically compared inhibitors of the MAPK and $\mathrm{PI} 3 \mathrm{~K}-\mathrm{AKT}$ signalling pathways, we observed subpopulations sensitive to both CRAF, ERK, or RSK targeted drugs and other drugs targeting the PI3K-AKT pathway; however, there were few instances of these subpopulations for inhibitors targeting other genes in the MAPK signalling. ${ }^{39}$ We found many more subpopulations that were more sensitive to BRAF inhibitors than other PI3KAKT inhibitors, and as expected, many contained BRAF mutations. $^{34}$ In contrast, there were not significantly more subpopulations sensitive to MEK inhibition compared to inhibition of PI3K-AKT signalling targets, but BRAF mutant subpopulations may have greater differential response. ${ }^{14}$ Divergent response was observed when comparing EGFR, BRAF, and MEK inhibitors to drugs targeting the PI3K-AKT pathway. This unique type of response was where subpopulations were found to be sensitive to either drug but not both. Our results comparing the MAPK and PI3K-AKT pathways based on drug response profiles highlights how intertwined those two pathways are in pharmacology space. $^{39}$

Arguably, the divergent response type is the most exciting for personalised treatment, since it may identify cases where independent drug action and synergy may guide effective drug combinations. ${ }^{21}$ Here exemplified, we showed that PI3K inhibitors combined with either BRAF or MEK inhibitors increase in vitro synergy and reduce tumour volume of in vivo models. Furthermore, we were able to show that synergistic and overall effect can be further enhanced by the correct biomarker indication, in this instance, BRAF mutant subpopulations. ${ }^{40,41}$ The BRAF mutant subpopulation with high efficacy for the BRAF inhibitor and not the other inhibitor could be cases where independent drug action explains drug combination efficacy, whereas the subpopulation with lower efficacy for single treatments of either drug may be cases for synergistic effects when the drugs are combined.

In examining the preclinical evidence for trial testing combination treatment of NSCLC in which the KRAS mutation was the biomarker, ${ }^{30}$ SEABED revealed a high proportion of NSCLC 
subpopulations having the KRAS mutation that are resistant to both selumetinib and docetaxel, suggesting a smaller likelihood of efficacy for the drug combination. Alternately, we identified a subpopulation with differential response to selumetinib for a small proportion of KRAS NSCLC cell lines, but this subpopulation contained a higher proportion of colorectal and pancreatic cancer cells with KRAS mutations. Previous studies have shown the plausibility in treating colorectal cancer using MEK inhibitor combinations. ${ }^{42,43}$ With consideration of KRAS mutations in subpopulations having greater sensitivity to selumetinib, SEABED suggests that while the correct biomarker was used for the clinical trial, there may be other potential indications for selumetinib. Future clinical trials testing MEK inhibitors in colorectal and pancreatic cancer could select patients possessing KRAS mutations to improve their chances of success. Although response in cell lines may not always correspond to response clinically, the use of data-informed approaches to examine large populations of cells may reveal clinically relevant drug response patterns. Future studies may need to account for differences between in vitro and in vivo responses.

While iterative and hierarchical clustering techniques have been used widely to attribute molecular markers to differences in subpopulation drug response and outcomes, ${ }^{44,45}$ we use an approach that does not require an explicit estimate of the number of subpopulations and is not greedy, i.e., each incremental step is optimal but the overall algorithm is not. In biomedical data processing, there has been substantial concern, particularly regarding applications to molecular data, that rival unsupervised machine learning methods optimise different criteria and consequently yield diverging answers. ${ }^{46,47}$ In our effort, we are concerned with discovering subpopulations having high homogeneity and statistical separability, while avoiding subpopulations that are so small that extracting statistically significant biomarkers is unlikely. We demonstrated the utility of SEABED over conventional approaches of K-Means and hierarchical clustering for drug response comparisons (see Supplementary Materials, Supplementary Tables S2 and S3, and Supplementary Fig. S6). Further, the ability to segment non-convex regions, which can arise to describe different disease states, is advantageous. Consequently, because of their success in other industries ${ }^{48,49}$ as well as fast, efficient implementations, spectral methods based on network models are powerful methods for discovering distinct subpopulations. $^{50-52}$

SEABED builds upon previous work using network models in biomedical contexts $5^{50,51,53}$ that explicitly partition a population of cell lines described by multiple variables into distinct subpopulations, using a "top-down" approach of recursively identifying optimal cuts for graph bisection. Similarly, while our segmentation capitalises on past progress made in spectral clustering, ${ }^{54,55}$ our effort distinguishes itself from past attempts by integrating all variables into a single network model using a multivariate similarity and local and global network statistics. Deeper interpretations of embedded matrix subspaces in network models may provide further insight into the linkage between subpopulations of cancer cell lines and drugs.

As a whole, this study demonstrates several important insights about the pharmacological pattern of response for different cancer drugs by applying an unsupervised machine learning platform to segment a large pan-cancer in vitro pharmacology data set. By organising cell lines along similar pharmacological patterns of response, we identified distinct, intrinsic subpopulations sensitive to one drug but resistant to others, and in some cases identified genetic alterations that can be used as biomarkers for those subpopulations. Since these genetic alterations are often driver mutations found in patient tumours, ${ }^{4}$ we can use these to map new patients recruited into clinical studies to our subpopulations derived from cell lines and predict their differential treatment response to existing treatments. In the context of analytical frameworks for increasing drug $R \& D$ productivity by sharpening the focus of drugs, ${ }^{56}$ our work demonstrates the value of advanced analytical approaches in translational medicine to enable decision making that is more data-informed and less ambiguous. Moreover, by analyzing different pharmacological responses and interpreting its outputs in the context of the underlying genetics and molecular pathways, we have created a multi-faceted landscape for developing and assessing new drug therapies.

\section{METHODS}

\section{Pharmacology data}

The discovery pharmacology dataset was extracted from the GDSC database, ${ }^{3,4}$ while leads from the analysis were validated with the Cancer Cell Line Encyclopaedia (CCLE) $)^{5}$ and the Cancer Therapeutics Response Portal (CTRP). ${ }^{6-8}$ Furthermore, suggested drug combinations were validated with cell line responses from the AstraZeneca-DREAM challenge dataset $^{25}$ and patient-derived xenograft (PDX) models from Gao et al. ${ }^{26}$ Drug synergy was defined as the total efficacy of two drugs that is greater than the individual effects of each drug. Combenefit (v1.31) was used to quantify drug combination synergy by comparing the observed twodimensional (2-D) combination dose-response surface to a modelled reference based on the Loewe model of additive combinations and the single drug dose-response curves. ${ }^{57}$

For a given cell line in GDSC, the drug response was fitted with a sigmoid curve ${ }^{58}$ and consecutively quantified as area under the curve (AUC) or the concentration required to reduce cell viability by half $\left(I_{50}\right)$. GDSC contains 265 compounds tested in 1074 cell lines, while we focus on a subset of 38 drugs targeting either PI3K-AKT signalling, MAPK signalling, or microtubules, which leads to 327 experiments considered for evaluation.

\section{Deep molecular characterisation of the cancer cell lines}

The GDSC project ${ }^{4}$ provides the characterisation of $>1000$ cell lines including whole-exome sequencing, targeted PCR sequencing/split probe FISH analysis and SNP6.0 arrays, which enabled to quantify somatic mutations, gene fusions, and copy number variations (CNVs), respectively. In our analysis, we focus on somatic mutational state of 300 cancer genes and 10 gene fusions. Additionally, we considered 425 recurrent CNVs, split into 117 amplifications and 308 deletions. In total, we consider 735 cancer functional events, which is summarised in the binary event matrix (BEM) from lorio et al. ${ }^{4}$ The SPEED algorithm was applied to gene expression profiles in the cell lines to quantify pathway activation as binary scores and then added to the BEM. ${ }^{59}$

\section{Pre-processing cell line and pharmacological data}

For every pair of drugs that was computationally analysed, the subset of GDSC cell lines having valid $\mathrm{IC}_{50}$ and AUC values for both drugs was retained. Typically there were roughly 700 cell lines across all cancer types that met these criteria in each experiment. Cancer types and subtypes were stored along with the pharmacological data in a table for each drug for subsequent recall and analysis.

\section{Processing drug response measures ( $A \cup C / \mathrm{IC}_{50}$ values)}

We build network models for a set of $N$ cell lines, $C=\left\{C_{1}, . ., C_{N}\right\}$, which are separately exposed to two distinct drugs, $D_{1}$ and $D_{2}$, which results in two sets of $M$ measurement variables, $\mathbf{X}_{\mathbf{i}}=\left[x_{1}, \ldots, x_{M}\right], i=1,2$, describing the response to each compound:

$\mathbf{X}_{\mathrm{i}, \mathrm{j}}=D_{i}\left(C_{j}\right), i=1,2 ; j=1, \ldots, N$

We use a network model that is an undirected graph, $G$, consisting of $N$ vertices, $V_{i}, i=1, \ldots, N$ (one for each cell line in $C$ ) with weighted edges, $W_{i, j}\left(V_{i}, V_{j}\right), i, j=1, \ldots, N, i \neq j$, between every distinct pair of vertices. Our approach uses a single multivariate similarity measure (Eq. 2), to construct one network model, with the advantage that the subspace properties of the resulting adjacency and Laplacian matrices are fully embedded with the complete characteristics of $C$. The weight is the similarity, $w_{i, j}$, between ith and $j$ th composite $2 M x 1$ dose response profile (DRP), $\mathbf{X}_{i}=\left[\mathbf{X}_{1, i}, \mathbf{X}_{2, i}\right]$ for $C_{i}$ and $\mathbf{X}_{j}=\left[\mathbf{X}_{1, j}, \mathbf{X}_{2, j}\right]$ for $C_{j}$.

We characterise drug response by two important continuous-valued measurements extrapolated from the cell line pharmacology screens: the 
$I_{50}$ and the AUC values of the dose-response curve (Supplementary Table S4) observed when one compound is applied in vitro to a single cell line sample at successively greater concentrations. Since every cell line possesses a length-4 DRP for a given pair of drugs, the similarity, $w_{\text {, }}$ between any two cell lines resides on $(0,1]$ and is calculated by a multivariate quasi-Gaussian comparison that differences the elements of the DRPs but also weighs the differences by a combination of local and global network statistics. Similarity between the response vectors, $\mathbf{X}_{i}$ and $\mathbf{X}_{j}$, is given by

$w\left(\mathbf{X}_{i}, \mathbf{X}_{j}\right)=\exp \left(-d\left(\mathbf{X}_{i}, \mathbf{X}_{j}\right)\right)$,

$d\left(\mathbf{X}_{i}, \mathbf{X}_{j}\right)=\left(\Delta \mathbf{X}_{i, j}^{\top} \Sigma_{i, j}^{-1} \Delta \mathbf{X}_{i, j}\right)^{\beta}$.

The similarity between two cell lines equals one when both have identical covariate values, and approaches zero as their covariates increasingly differ. Additionally, $w\left(\mathbf{X}_{i}, \mathbf{X}_{i}\right)=w\left(\mathbf{X}_{j}, \mathbf{X}_{i}\right) . \Delta \mathbf{X}_{i, j}$ is a $4 \times 1$ vector whose entries are the difference of the DRP values in $\mathbf{X}_{i}$ and $\mathbf{X}_{j}$ and $\beta$ modulates the similarity between two patients. We selected $\beta=0.5$ for our experiments based on experimentation and the observations of previous efforts.

$\Sigma_{i, j}$ is a $4 \times 4$ covariance-like matrix that is estimated for every distinct ( $i$, j)-pair and captures the variability of individual variables as well as their inter-relationships. While $\Sigma_{i, j}$ is an explicit function of the two patients being compared, it also captures network-wide characteristics. For diagonal elements, $\Sigma_{i, j}(a, a), a=1, \ldots 4$, the entries are:

$\Sigma_{i, j}(a, a)=\frac{\left(\Delta X_{i, j}(a)+\underline{\operatorname{Ngbd}\left(\Delta X_{l}(a)\right)}++\underline{\operatorname{Ngbd}\left(\Delta X_{J}(a)\right)}\right)^{2}}{9}, a=1,2,3,4$

where $\operatorname{Ngbd}\left(\Delta X_{l}(a)\right)$ corresponds to all edges neighbouring vertex-i, and the overbar is the averaging operator. The off-diagonal elements, $\Sigma_{i, j}(a, b), a, b=1, . .4, a \neq b$ are:

$\Sigma_{i j}(a, b)$

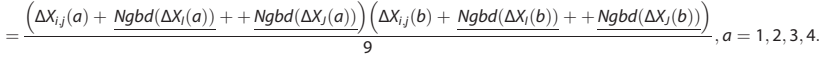

The Moore-Penrose pseudo-inverse was used to avoid problems with low-rank during matrix inversion. The framework is generalisable to include more variables and different measures of similarity. The symmeric, positive semi-definite, $N \times N$ weighted adjacency matrix, $\mathbf{W}$, holds the pairwise similarities.

\section{Segmentation}

The set of cell lines, $C$, is segmented recursively into distinct subpopulations using the Fiedler eigenvector derived from the eigendecomposition of $\mathbf{W}{ }^{60}$ Each parent subpopulation of cell lines is successively segmented into two offspring subpopulations until at least one of three constraints is satisfied:

- The size of the parent subpopulation falls below a user-defined threshold.

- The size of either offspring subpopulation is below a user-defined threshold.

- The offspring subpopulations are not sufficiently dissimilar, where we measure class separability using the silhouette metric, ${ }^{61}$ a common non-parametric method in which values range between -1 (highly similar) and 1 (highly dissimilar) and values near 0 indicate the two subpopulations are just barely overlapping. Higher values result in fewer, less granular, subpopulations.

In our experiments, we required the size of the parent subpopulation to be at least 40 in order for segmentation to be performed, and required both offspring subpopulations to have 20 or more members in order to be retained. The silhouette metric threshold was set to 0.25 . Generally, criteria and thresholds can be modified and adapted to emphasise relevant factors in a particular problem. The whole segmentation process yields $K$ mutually exclusive subpopulations $P_{k}, k=1, \ldots, K$, where $C=\cup_{k=1}^{K} P_{k}$. Successive segmentation results in sub-populations with increasingly homogeneous DRPs.

\section{Enrichment of features to nominate biomarkers}

Because genetic alterations in each cell line are known, each subpopulation can be evaluated by non-parametric statistical tests to identify enriched alterations that may be attributed to patterns of sensitivity or resistance in the DRP across both drugs. For each subpopulation, we measured the number of cell lines in the subpopulation with a particular gene mutation, and the number of cell lines outside of the subpopulation with the mutation. A $2 \times 2$ contingency table was generated from the cell line counts of with/without mutation and inside/outside of subpopulation. Significance of observed enrichment of mutations within subpopulations were calculated using the Fisher's exact test. The resulting $p$ values were corrected for multiple testing using the Benjamini and Hochberg $(\mathrm{BH})$ procedure (Supplementary Table S5).

\section{Classification of drug pairs based on the distribution of subpopulations}

We made 324 pairwise comparisons of drugs targeting the MAPK and PI3K-AKT pathways. Based on the distribution of $\log \left({ }\left(C_{50}\right)\right.$ values across all cell lines tested with both drugs, we determined the 20th-percentile of log $\left(I_{50}\right)$ values for each drug to be a user-defined cutoff. The 20th percentile cutoffs $P_{20}$ for drugs $\mathrm{A}$ and $\mathrm{B}$ was used to categorise each subpopulation $i$ into four categories based on their average $\log \left(I C_{50}\right) y$ :

$y_{i}<P_{20, A}$ and $y_{i}<P_{20, B}=$ sensitive to drugs $A$ and $B$

$y_{i}<P_{20, A}$ and $y_{i} \geq P_{20, B}=$ more sensitive to drug $A$

$y_{i} \geq P_{20, A}$ and $y_{i}<P_{20, B}=$ more sensitive to drug $B$

$y_{i} \geq P_{20}, A$ and $y_{i} \geq P_{20, B}=$ resistant to drugs $A$ and $B$

The number of subpopulations in each category were recorded in a $2 \times 2$ contingency matrix and using a binomial test compared this to the proportion of cells expected in each category if SEABED segmentation was not performed.

After classification of pairwise drug responses, we assessed whether a drug's subpopulations was significantly over-represented in one category in comparison with all other categories. Testing was carried out using the hypergeometric test (phyper R package).

\section{2-D visualisation of drug response profiles}

To visualise DRPs across cell lines and drug comparisons, we calculated the average $\log \left(I_{50}\right)$ values for each drug in subpopulations generated based on their response to the tested drug pairs (Supplementary Table S5). We then plotted the mean $\log \left(\mathrm{IC}_{50}\right)$ values as circles on a 2-D scatter plot using the Matplotlib Python library. Dashed lines indicative of local 20th percentile of $\log \left(I_{50}\right)$ values for each drug were also plotted on the scatter plot unless stated otherwise. The radii of the circles is proportional to the subpopulation size. Due to potential misidentification, cell lines named 'NA' found in the output files after segmentation with SEABED were not used for the calculation of the mean $\log \left(I_{C_{50}}\right)$ values of each subpopulation and, hence, not used for the 2-D visualisation. Sensitivity cutoffs for each drug assumed that $20 \%$ of cell lines had an $\mathrm{IC}_{50}$ beyond an arbitrary threshold, which was a consensus among drug development scientists and a lower threshold for clinically approved drugs. ${ }^{62}$ These human-defined cutoffs were used as a guide for comparing our algorithmically segmented subpopulations.

\section{Tree visualisation of subpopulations}

We utilised tree diagrams to visualise the data generated. The tree diagrams illustrate how the cancer cell lines are segmented into different subpopulations, based on whether they are sensitive or resistant to the drugs that are being tested. We did not visualise further down the tree in the figures when previously observed significant enrichment of genetic biomarkers is no longer observed in all current subpopulations. The tree diagrams were generated through an open-source Python library called Graphviz. The style of each component of the tree diagram was first initialised through a class. This included the colours, shapes, and fonts of the edges and nodes of the tree diagram. A method to create tree diagrams was developed to accept the number of vertices and leaves, the labels for the leaves, and the tree diagram filename. The tree diagram is finally generated and saved by calling the method.

\section{Reporting Summary}

Further information on research design is available in the Nature Research Reporting Summary linked to this article. 


\section{DATA AVAILABILITY}

All data used in the paper are published previously and publicly available at the GDSC (https://www.cancerrxgene.org/), CCLE (https://portals.broadinstitute.org/ccle), and CTRP (https://portals.broadinstitute.org/ctrp/) databases. Datasets used are listed in Supplementary Table S4, Supplementary Table S5, and the Key Resources Table.

\section{CODE AVAILABILITY}

All code for the pipeline is open source and available at: https://github.com/szen95/ SEABED. Further information and requests should be directed to and will be fulfilled by the co-corresponding authors, Michael P. Menden (michael.menden@helmholtzmuenchen.de) or Dennis Wang (dennis.wang@sheffield.ac.uk).

Received: 28 April 2019; Accepted: 5 September 2019; Published online: 03 October 2019

\section{REFERENCES}

1. Weinstein, J. N. Cell lines battle cancer. Nature 483, 544-545 (2012).

2. Shoemaker, R. H. The $\mathrm{NCl} 60$ human tumour cell line anticancer drug screen. Nat. Rev. Cancer 6, 813-823 (2006).

3. Garnett, M. J. et al. Systematic identification of genomic markers of drug sensitivity in cancer cells. Nature 483, 570-575 (2012).

4. Iorio, F. et al. A landscape of pharmacogenomic interactions in cancer. Cell 166, 740-754 (2016).

5. Barretina, J. et al. The Cancer Cell Line Encyclopedia enables predictive modelling of anticancer drug sensitivity. Nature 483, 603-607 (2012).

6. Rees, M. G. et al. Correlating chemical sensitivity and basal gene expression reveals mechanism of action. Nat. Chem. Biol. 12, 109-116 (2016).

7. Seashore-Ludlow, B. et al. Harnessing connectivity in a large-scale small-molecule sensitivity dataset. Cancer Discov. 5, 1210-1223 (2015).

8. Basu, A. et al. An interactive resource to identify cancer genetic and lineage dependencies targeted by small molecules. Cell 154, 1151-1161 (2013).

9. Yang, W. et al. Genomics of Drug Sensitivity in Cancer (GDSC): a resource for therapeutic biomarker discovery in cancer cells. Nucleic Acids Res. 41, D955-61 (2013).

10. Silverbush, D. et al. Cell-specific computational modeling of the PIM pathway in acute myeloid leukemia. Cancer Res. 77, 827-838 (2017).

11. Costello, J. C. et al. A community effort to assess and improve drug sensitivity prediction algorithms. Nat. Biotechnol. 32, 1202-1212 (2014).

12. Ravikumar, B., Alam, Z., Peddinti, G. \& Aittokallio, T. C-SPADE: a web-tool for interactive analysis and visualization of drug screening experiments through compound-specific bioactivity dendrograms. Nucleic Acids Res. 45, W495-W500 (2017).

13. Patel, S. P. et al. Clinical responses to selumetinib (AZD6244; ARRY-142886)-based combination therapy stratified by gene mutations in patients with metastatic melanoma. Cancer 119, 799-805 (2013).

14. Catalanotti, F. et al. Phase II trial of MEK inhibitor selumetinib (AZD6244, ARRY142886) in patients with BRAFV600E/K-mutated melanoma. Clin. Cancer Res. 19, 2257-2264 (2013).

15. Metro, G. et al. Selumetinib: a promising pharmacologic approach for KRASmutant advanced non-small-cell lung cancer. Future Oncol. 9, 167-177 (2013).

16. Kim, D. W. \& Patel, S. P. Profile of selumetinib and its potential in the treatment of melanoma. Onco Targets Ther. 7, 1631-1639 (2014).

17. Tricker, E. M. et al. Combined EGFR/MEK inhibition prevents the emergence of resistance in EGFR-mutant lung cancer. Cancer Discov. 5, 960-971 (2015).

18. Sequist, L. V. et al. Phase III study of Afatinib or Cisplatin plus pemetrexed in patients with metastatic lung adenocarcinoma with EGFR mutations. J. Clin. Oncol. 31, 3327-3334 (2013)

19. Misale, S. et al. Emergence of KRAS mutations and acquired resistance to antiEGFR therapy in colorectal cancer. Nature 486, 532-536 (2012).

20. Geyer, C. E. et al. Lapatinib plus capecitabine for HER2-positive advanced breast cancer. N. Engl. J. Med. 355, 2733-2743 (2006).

21. Palmer, A. C. \& Sorger, P. K. Combination cancer therapy can confer benefit via patient-to-patient variability without drug additivity or synergy. Cell 171, 1678-1691.e13 (2017).

22. Fujishita, T., Kajino-Sakamoto, R., Kojima, Y., Taketo, M. M. \& Aoki, M. Antitumor activity of the MEK inhibitor trametinib on intestinal polyp formation in Apc $(\Delta 716)$ mice involves stromal COX-2. Cancer Sci. 106, 692-699 (2015).

23. De Grève, J. et al. Clinical activity of afatinib (BIBW 2992) in patients with lung adenocarcinoma with mutations in the kinase domain of HER2/neu. Lung Cancer 76, 123-127 (2012).
24. Jacobsen, K. et al. Convergent Akt activation drives acquired EGFR inhibitor resistance in lung cancer. Nat. Commun. 8, 410 (2017).

25. Menden, M. P. et al. Community assessment to advance computational prediction of cancer drug combinations in a pharmacogenomic screen. Nat. Commun. 10, 2674 (2019).

26. Gao, H. et al. High-throughput screening using patient-derived tumor xenografts to predict clinical trial drug response. Nat. Med. 21, 1318-1325 (2015).

27. Martin-Liberal, J. Encorafenib plus binimetinib: an embarrassment of riches. Lancet Oncol. 19, 1263-1264 (2018).

28. Dummer, R. et al. Overall survival in patients with BRAF-mutant melanoma receiving encorafenib plus binimetinib versus vemurafenib or encorafenib (COLUMBUS): a multicentre, open-label, randomised, phase 3 trial. Lancet Oncol. 19, 1315-1327 (2018).

29. Paller, C. J. et al. Design of phase I combination trials: recommendations of the clinical trial design task force of the $\mathrm{NCl}$ Investigational Drug Steering Committee. Clin. Cancer Res. 20, 4210-4217 (2014).

30. Jänne, P. A. et al. Selumetinib plus docetaxel compared with docetaxel alone and progression-free survival in patients with KRAS-mutant advanced non-small cell lung cancer. JAMA 317, 1844 (2017).

31. Garon, E. B. et al. Identification of common predictive markers of in vitro response to the Mek inhibitor selumetinib (AZD6244; ARRY-142886) in human breast cancer and non-small cell lung cancer cell lines. Mol. Cancer Ther. 9, 1985-1994 (2010).

32. Long, G. V. et al. Combined BRAF and MEK inhibition versus BRAF inhibition alone in melanoma. N. Engl. J. Med. 371, 1877-1888 (2014).

33. Van Allen, E. M. et al. The genetic landscape of clinical resistance to RAF inhibition in metastatic melanoma. Cancer Discov. 4, 94-109 (2014).

34. Holderfield, M., Deuker, M. M., McCormick, F. \& McMahon, M. Targeting RAF kinases for cancer therapy: BRAF-mutated melanoma and beyond. Nat. Rev. Cancer 14, 455-467 (2014).

35. Hall, R. D. \& Kudchadkar, R. R. BRAF mutations: signaling, epidemiology, and clinical experience in multiple malignancies. Cancer Control 21, 221-230 (2014).

36. Abdel-Wahab, O. et al. Efficacy of intermittent combined RAF and MEK inhibition in a patient with concurrent BRAF- and NRAS-mutant malignancies. Cancer Discov. 4, 538-545 (2014).

37. Paz-Ares, L. et al. Afatinib versus gefitinib in patients with EGFR mutation-positive advanced non-small-cell lung cancer: overall survival data from the phase $\mathrm{llb}$ LUX-Lung 7 trial. Ann. Oncol. 28, 270-277 (2017).

38. Takezawa, K. et al. HER2 amplification: a potential mechanism of acquired resistance to EGFR inhibition in EGFR-mutant lung cancers that lack the secondsite EGFRT790M mutation. Cancer Discov. 2, 922-933 (2012).

39. Mendoza, M. C., Er, E. E. \& Blenis, J. The Ras-ERK and PI3K-mTOR pathways: crosstalk and compensation. Trends Biochem. Sci. 36, 320-328 (2011).

40. Temraz, S., Mukherji, D. \& Shamseddine, A. Dual inhibition of MEK and PI3K pathway in KRAS and BRAF mutated colorectal cancers. Int. J. Mol. Sci. 16, 22976-22988 (2015).

41. Villanueva, J., Vultur, A. \& Herlyn, M. Resistance to BRAF inhibitors: unraveling mechanisms and future treatment options. Cancer Res. 71, 7137-7140 (2011).

42. Ebert, P. J. R. et al. MAP kinase inhibition promotes T cell and anti-tumor activity in combination with PD-L1 checkpoint blockade. Immunity 44, 609-621 (2016).

43. Bendell, J. et al. LBA-01 safety and efficacy of cobimetinib (cobi) and atezolizumab (atezo) in a Phase 1b study of metastatic colorectal cancer (mCRC). Ann. Oncol. 27, ii140-ii140 (2016).

44. Gholami, A. M. et al. Global proteome analysis of the $\mathrm{NCl}-60$ cell line panel. Cell Rep. 4, 609-620 (2013).

45. Eisen, M. B., Spellman, P. T., Brown, P. O. \& Botstein, D. Cluster analysis and display of genome-wide expression patterns. Proc. Natl Acad. Sci. USA 95, 14863-14868 (1998).

46. Wiwie, C., Baumbach, J. \& Röttger, R. Comparing the performance of biomedical clustering methods. Nat. Methods 12, 1033-1038 (2015).

47. Monti, S., Tamayo, P., Mesirov, J. \& Golub, T. Consensus clustering: a resamplingbased method for class discovery and visualization of gene expression microarray data. Mach. Learn. 52, 91-118 (2003)

48. Sarwar, B., Karypis, G., Konstan, J. \& Reidl, J. Item-based collaborative filtering recommendation algorithms. In Proc. Tenth International Conference on World Wide Web-WWW'01 (Hong Kong, 2001).

49. Scott, J. Social Network Analysis (SAGE, 2017).

50. Wang, B. et al. Similarity network fusion for aggregating data types on a genomic scale. Nat. Methods 11, 333-337 (2014).

51. Li, L. et al. Identification of type 2 diabetes subgroups through topological analysis of patient similarity. Sci. Transl. Med. 7, 311ra174 (2015).

52. Nicolau, M., Levine, A. J. \& Carlsson, G. Topology based data analysis identifies a subgroup of breast cancers with a unique mutational profile and excellent survival. Proc. Natl Acad. Sci. USA 108, 7265-7270 (2011). 
53. Kim, D. et al. Knowledge boosting: a graph-based integration approach with multi-omics data and genomic knowledge for cancer clinical outcome prediction. J. Am. Med. Inform. Assoc. https://doi.org/10.1136/amiajnl-2013002481 (2014).

54. Ng, A. Y., Jordan, M. I. \& Weiss, Y. On spectral clustering: Analysis and an algorithm. in Advances in Neural Information Processing Systems (2002).

55. Shi, J. \& Malik, J. Normalized cuts and image segmentation. IEEE Trans. Pattern Anal.Mach. Intell 22, 888-905 (2000).

56. Cook, D. et al. Lessons learned from the fate of AstraZeneca's drug pipeline: a five-dimensional framework. Nat. Rev. Drug Discov. 13, 419-431 (2014).

57. Di Veroli, G. Y. et al. Combenefit: an interactive platform for the analysis and visualization of drug combinations. Bioinformatics 32, 2866-2868 (2016).

58. Vis, D. J. et al. Multilevel models improve precision and speed of IC50 estimates. Pharmacogenomics 17, 691-700 (2016).

59. Parikh, J. R., Klinger, B., Xia, Y., Marto, J. A. \& Blüthgen, N. Discovering causal signaling pathways through gene-expression patterns. Nucleic Acids Res. 38, W109-17 (2010).

60. Fiedler, M. A property of eigenvectors of nonnegative symmetric matrices and its application to graph theory. Czechoslovak Math. J. 25, 619-633 (1975).

61. Rousseeuw, P. J. Silhouettes: a graphical aid to the interpretation and validation of cluster analysis. J. Comput. Appl. Math. 20, 53-65 (1987).

62. Chen, E. Y., Raghunathan, V. \& Prasad, V. An overview of cancer drugs approved by the US Food and Drug Administration based on the surrogate end point of response rate. JAMA Intern. Med. 179, 915-921 (2019).

\section{ACKNOWLEDGEMENTS}

We would like to thank Ben Sidders (AstraZeneca plc.), Jonathan Dry (AstraZeneca plc.), Francesco lorio (Sanger Institute), Michael Schubert (EMBL-EBI), Mi Yang (RWTH Aachen), and Winston Hide (Harvard University) for useful discussions. This work is supported by funding from the NIHR Sheffield Biomedical Research Centre, Rosetrees Trust (ref: A2501), and the Academy of Medical Sciences Springboard (ref: SBF004/ 1052).

\section{AUTHOR CONTRIBUTIONS}

N.K., T.S.T., M.P.M., and D.W. contributed to the conceptualisation of the project. N.K. T.S.T., and D.W. were responsible for data curation. Formal analysis was performed by N.K., T.S.T., H.Y., B.Y., M.P.M., and D.W. M.M. and D.W. were responsible for funding acquisition. The methodology was developed by N.K., T.S.T., M.P.M., and D.W. T.S.T. and D.W. were responsible for administration of the project. Resources for the experiments were prepared by D.W. and N.K. Various software for the project was developed and implemented by N.K., T.S.T., H.Y., and D.W. M.P.M and D.W. oversaw supervision of the project. N.K., T.S.T., M.P.M., and D.W. wrote the manuscript.

\section{COMPETING INTERESTS}

NK is an employee of Constellation Analytics, LLC. The remaining authors declare no competing interests.

\section{ADDITIONAL INFORMATION}

Supplementary information is available for this paper at https://doi.org/10.1038/ s41540-019-0113-4.

Correspondence and requests for materials should be addressed to M.P.M., D.W.

Reprints and permission information is available at http://www.nature.com/ reprints

Publisher's note Springer Nature remains neutral with regard to jurisdictional claims in published maps and institutional affiliations. (c) (i)

Open Access This article is licensed under a Creative Commons Attribution 4.0 International License, which permits use, sharing, adaptation, distribution and reproduction in any medium or format, as long as you give appropriate credit to the original author(s) and the source, provide a link to the Creative Commons license, and indicate if changes were made. The images or other third party material in this article are included in the article's Creative Commons license, unless indicated otherwise in a credit line to the material. If material is not included in the article's Creative Commons license and your intended use is not permitted by statutory regulation or exceeds the permitted use, you will need to obtain permission directly from the copyright holder. To view a copy of this license, visit http://creativecommons. org/licenses/by/4.0/.

(c) The Author(s) 2019 